\title{
Inercia terapéutica del médico familiar en pacientes con descontrol hipertensivo.
}

DOI: $10.46932 / \mathrm{sfjdv} 1 \mathrm{n} 4-008$

Received in: August 1st, 2020

Accepted in: September 30th, 2020

\section{José Felíx Mendoza González}

Higher academic background: Médico Familiar

Current Institution: Servicio de Atención Familiar. Unidad Médica Familiar 12, Tamasopo San Luis Potosí, Instituto Mexicano del Seguro Social, México.

Full address (can be institutional or personal, as you prefer): Kilómetro 2, carretera Tamasopo Agua buena, San Luis Potosí, CP. 79700.

E-mail: p_pox@hotmail.com

\section{Jesús Javier Mejía Inglés}

Higher academic background: Médico Familiar

Current Institution: Jefatura de Departamento Clínico en Atención Familiar. Unidad Médica Familiar 77, Madero Tamaulipas, Instituto Mexicano del Seguro Social, México.

Full address (can be institutional or personal, as you prefer): Blvd. Adolfo López Mateos S/N, Col. Las Conchitas, Madero Tamaulipas.

E-mail: jesadry.28@gmail.com

\section{Liliana Aurora Carrillo Aguiar}

Higher academic background: Médico Familiar., Master en Ciencias Médicas., Profesor Titular de la Residencia en Medicina Familiar.

Current Institution: Unidad Médica Familiar 77, Madero Tamaulipas, Instituto Mexicano del Seguro Social, México.

Full address (can be institutional or personal, as you prefer): Blvd. Adolfo López Mateos S/N, Col. Las

Conchitas, Madero Tamaulipas.

E-mail: lilifha@hotmail.com

\section{Brian González Pérez}

Higher academic background: Médico Familiar., Master en Ciencias Médicas., Master en Innovación y Tecnología Educativa., Profesor Investigador.

Current Institution: Servicio de Atención Familiar. Unidad Médica Familiar 38, Tampico Tamaulipas, Instituto Mexicano del Seguro Social, México. Facultad de Medicina "Dr. Alberto Romo Caballero"

Universidad Autónoma de Tamaulipas, Tampico, Tamaulipas, México.

Full address (can be institutional or personal, as you prefer): Calle Argentina y Canadá $107 \mathrm{Col}$.

Infonavit C.P. 89310, Tampico, Tamaulipas.

E-mail: brian.gonzalez.perez@gmail.com 


\section{Ricardo Salas Flores \\ Higher academic background: Endocrinólogo Pediatra., Master en Ciencias Médicas., Profesor Investigador.}

Current Institution: Coordinación de Educación en Salud e Investigación. Hospital General Regional No. 6, Madero, Tamaulipas, Instituto Mexicano del Seguro Social, México. Facultad de Medicina "Dr.

Alberto Romo Caballero" Universidad Autónoma de Tamaulipas, Tampico, Tamaulipas, México. Full address (can be institutional or personal, as you prefer): Blvd. Adolfo López Mateos S/N, Col. Las

Conchitas, Madero Tamaulipas.

E-mail: risafl@yahoo.com

\section{RESUMEN}

INTRODUCCION: En el manejo de los pacientes portadores de hipertensión arterial, la inercia terapéutica se define como la reticencia de los médicos familiares a optimizar la terapia farmacológica y no lograr los objetivos de control de presión arterial siendo una de las principales causas del descontrol y de aparición de complicaciones cardiovasculares.

OBJETIVO: Conocer la inercia terapéutica del médico familiar en el manejo de pacientes con hipertensión arterial sistémica descontrolada de la Unidad de Medicina Familiar No 77.

METODOS: Se realizó un estudio observacional, descriptivo, transversal y retrospectivo, en pacientes de ambos sexos, de edades entre los 18 y 79 años de edad con hipertensión arterial descontrolada de enero a marzo 2018, donde se evaluaron las características de las determinaciones de presión arterial e intervenciones terapéuticas del médico familiar registradas en el expediente clínico. Se incluyeron las variables edad, género, presión arterial sistólica, diastólica, índice de masa corporal, cambios en el tratamiento, ajuste de medicamentos y años de evolución. La inercia terapéutica se cuantificó como el número de pacientes a los que no se les modificó el tratamiento farmacológico, dividido entre el número de pacientes que no han conseguido el objetivo de control y multiplicado por 100, clasificándola como leve A $(<20 \%)$, moderada, B (20-50\%) y severa C (>50\%). Se aplicaron estadísticas descriptivas, medidas de tendencia central y análisis de asociación entre variables cualitativas mediante la prueba de $\mathrm{X}^{2}$. Un valor de $\mathrm{p}<0.05$ fue considerado como significativo.

RESULTADOS: Se estudiaron un total de 150 casos de pacientes con hipertensión arterial descontrolada, el $56 \%(n=84)$ fueron femeninos y $44 \%(n=66)$ masculinos. La edad cronológica promedio de la población estudiada fue de $61.6 \pm 8.6$ años. El promedio de IMC fue $28.3 \pm 3.7$, de sistólica $127 \pm 10.1$, diastólica $79.3 \pm 6.2$ y para el tiempo de evolución de la enfermedad $8.5 \pm 4.4$ años. Los fármacos prescritos fueron diuréticos $28.3 \%$, seguido de los calcio-antagonistas con $19.7 \%$, IECA $18.5 \%$, ARA II $17.3 \%$ y en último lugar los beta-bloqueadores $16.2 \%$. En el $77.3 \%$ de los casos no presentaron inercia terapéutica y en 34 pacientes $(22.7 \%)$ se encontró una inercia terapéutica moderada $(>20 \%)$ asociada a la falta de cambios en el tratamiento ( $\mathrm{p}<0.001)$ y con los años de evolución de la presión arterial de $\mathrm{p}<0.002$.

CONCLUSIONES: La inercia terapéutica encontrada tiene una severidad moderada y es importante la capacitación del médico familiar en las guías de práctica clínica y la supervisión estricta de los pacientes con hipertensión descontrolada.

Palabras Clave: Inercia terapéutica, hipertensión arterial, médico familiar, atención médica, servicios de salud. 


\section{INTRODUCCION}

La hipertensión arterial sistémica es un síndrome de etiología múltiple caracterizado por la elevación persistente de las cifras de presión arterial a cifras $\geq 140 / 90 \mathrm{~mm} / \mathrm{Hg}$. Es considerada como un predictor de morbimortalidad para enfermedades cardiovasculares, entre las que destacan la enfermedad cerebrovascular, el infarto del miocardio, la insuficiencia cardiaca, la enfermedad arterial periférica y la insuficiencia renal (1). La hipertensión arterial causa anualmente 9.4 millones de muertes en el mundo y contribuye al $12.8 \%$ de la mortalidad por todas las causas (2). En los últimos años, su prevalencia en países de bajos ingresos ha sido de 40\% (3) y en México durante el año 2016 la prevalencia fue de $30.2 \%(4)$.

De acuerdo con la Encuesta Nacional de Salud y Nutrición de Medio Camino 2016, realizada por el Instituto Nacional de Salud Pública (INSP) y la Secretaría de Salud, uno de cada cuatro adultos en México padece hipertensión arterial, es decir, 25.5 por ciento de la población, de los cuales aproximadamente el 40 por ciento ignora que tiene esta enfermedad, y ello repercute en su condición de salud; y cerca del 60 por ciento que conoce el diagnóstico, solamente la mitad están controlados (5).

El diagnóstico oportuno, conocimiento de la enfermedad, acceso a fármacos antihipertensivos y servicios de salud de calidad son fundamentales para el control de la hipertensión (6). Sin embargo el hecho de no iniciar o intensificar el tratamiento de acuerdo con las guías de práctica clínica basadas en la evidencia, se reconoce cada vez más como un fenómeno que contribuye al manejo inadecuado de las condiciones crónicas y se conoce como inercia terapéutica (7). Por definición, la inercia terapéutica es la falla de un proveedor de servicios de salud para iniciar o intensificar la terapia farmacológica cuando no se han logrado metas de control óptimas (8).

En el caso concreto del manejo de los pacientes portadores de hipertensión arterial se define la inercia terapéutica, como la reticencia de los médicos familiares a optimizar la terapia farmacológica y no lograr los objetivos de control de presión arterial siendo una de las principales causas del descontrol y de aparición de complicaciones cardiovasculares (9). La principal manifestación de la inercia terapéutica es la falta de aumento de la dosis o número de medicamentos antihipertensivos para intensificar el tratamiento cuando la presión arterial aumenta o permanece por encima de los objetivos terapéuticos (10). Entre las principales causas descritas en la literatura causantes de mal control hipertensivo, destacan la metodología inadecuada de medida de la presión arterial, la falta de cumplimiento terapéutico de los pacientes y la inercia terapéutica de los médicos (11).

La inercia terapéutica depende muy especialmente de la formación y actitud del médico (12) y es ampliamente reconocida como una causa mayor del mal control de la hipertensión arterial y de otras enfermedades crónicas (13). Las repercusiones clínicas derivadas de la inercia terapéutica, que supone la 
no intensificación de las medidas higiénico-dietéticas o farmacológicas, incluye el retraso en el inicio del tratamiento y la falta de un correcto plan de seguimiento que garantice la detección e intervención ante complicaciones de la enfermedad (14).

Diferentes estudios realizados en nuestro país han aportado información indirecta sobre la magnitud actual del problema de la inercia terapéutica en la práctica clínica de atención primaria y especializada, en todos ellos se observó que la actitud terapéutica del médico ante el mal control de la presión arterial no es la recomendada por las guías de práctica clínica (15). Nuestro estudio permitirá conocer la inercia terapéutica del médico familiar en el manejo de pacientes con hipertensión arterial sistémica descontrolada de la Unidad de Medicina Familiar No 77.

\section{METODOS}

Se realizó un estudio observacional, transversal, descriptivo y retrospectivo en pacientes de ambos sexos, de edades entre los 18 y 79 años de edad con hipertensión arterial descontrolada que acudieron a la consulta externa de Medicina Familiar de la Unidad de Medicina Familiar No 77 en el periodo comprendido de enero a marzo 2018. Previo consentimiento informado, se evaluaron las características de las determinaciones de presión arterial e intervenciones terapéuticas del médico familiar registradas en el expediente clínico. Se incluyeron las variables edad, género, presión arterial sistólica, diastólica, índice de masa corporal (IMC), cambios en el tratamiento, ajuste de medicamentos y años de evolución. La inercia terapéutica se cuantificó como el número de pacientes a los que no se les modificó el tratamiento farmacológico, dividido entre el número de pacientes que no han conseguido el objetivo de control y multiplicado por 100, clasificándola como leve A (<20\%), moderada, B (20-50\%) y severa $\mathrm{C}(>50 \%)$. Los datos recolectados se analizaron con ayuda del paquete estadístico Statistical Package for Social Sciences para Windows (versión 19; SPSS Inc., Chicago IL, USA). Se aplicaron estadísticas descriptivas, medidas de tendencia central y análisis de asociación entre variables cualitativas mediante la prueba de X2. Un valor de $\mathrm{p}<0.05$ fue considerado como significativo. El protocolo de investigación fue aprobado por el Comité Local de Investigación en Salud 2804 del Instituto Mexicano del Seguro Social.

\section{RESULTADOS}

Se estudiaron un total de 150 casos de pacientes con hipertensión arterial descontrolada, el 56\% $(\mathrm{n}=84)$ fueron femeninos y 44\% $(\mathrm{n}=66)$ masculinos. La edad cronológica promedio de la población estudiada fue de $61.6 \pm 8.6$ años. El promedio de IMC fue $28.3 \pm 3.7$, de sistólica $127 \pm 10.1$, diastólica $79.3 \pm 6.2$ y para el tiempo de evolución de la enfermedad $8.5 \pm 4.4$ años. Los fármacos prescritos 
Tabla 1.- Características generales de la población estudiada

\begin{tabular}{|c|c|c|}
\hline \multirow{2}{*}{ Información general de los pacientes } & \multicolumn{2}{|c|}{$\begin{array}{l}\text { Hipertensión Arterial Descontrolada } \\
\qquad(n=150)\end{array}$} \\
\hline & $N$ & $\%$ \\
\hline \multicolumn{3}{|c|}{ Sexo } \\
\hline Hombres & 66 & 44 \\
\hline Mujeres & 84 & 56 \\
\hline $\mathrm{IMC}(\mathrm{kg} / \mathrm{m} 2) *$ & \multicolumn{2}{|c|}{$28.3+3.7$} \\
\hline Sistólica $(\mathrm{mmHg}) *$ & \multicolumn{2}{|c|}{$127 \pm 10.1$} \\
\hline Diastólica $(\mathrm{mmHg}) *$ & \multicolumn{2}{|c|}{$79.3 \pm 6.2$} \\
\hline Tiempo de evolución (años) $*$ & \multicolumn{2}{|c|}{$8.5 \pm 4.4$} \\
\hline \multicolumn{3}{|c|}{ Fármacos Prescritos } \\
\hline Diuréticos & 91 & 26.4 \\
\hline Calcio-antagonistas & 70 & 19.7 \\
\hline IECA & 68 & 18.5 \\
\hline ARA II & 59 & 17.4 \\
\hline Beta-bloqueadores & 58 & 16.2 \\
\hline \multicolumn{3}{|c|}{ Inercia Terapéutica $* *$} \\
\hline $\mathrm{Si}$ & 34 & 22.3 \\
\hline No & 116 & 77.3 \\
\hline
\end{tabular}

IECA (Inhibidores de la Enzima Convertidora de Angiotensina), ARA II (Antagonista de los receptores de angiotensina II).

* Valores expresados como $\square+\mathrm{DE}$

** Estadísticamente significativo $\mathrm{p}<0.05$

\section{DISCUSIÓN}

Para el control del paciente hipertenso, existe una gran variabilidad en el tratamiento farmacológico, en muchos casos, no se han instrumentado medidas no farmacológicas, y en un elevado porcentaje no existe un adecuado control de las cifras tensionales, con los consecuentes incrementos en el riesgo de daño en los órganos blanco, la discapacidad, los costos de la atención y su repercusión en la economía familiar, en los sistemas de salud y en el país (16). El manejo de pacientes con hipertensión arterial descontrolada por parte del médico familiar implica el riesgo de ocurrencia de la inercia terapéutica, sabiendo que existen barreras como una falsa impresión de buen control de la enfermedad, la percepción de mala adherencia del paciente y la falta de entrenamiento y organización. El instrumento para medir con exactitud la inercia terapéutica, basado en la definición operacional de $\mathrm{O}^{\prime}$ Connor et al, para poder establecer este fenómeno, permitió hacer la medición en todos los pacientes con hipertensión arterial sistémica no controlada (17). 
Estudios transversales multicéntricos indican que la magnitud de la Inercia terapéutica en el tratamiento de la hipertensión arterial en España y Latinoamérica oscila entre el 51 y $85 \%$ de los hipertensos no controlados (18), lo cual difiere con los resultados obtenidos en el presente estudio, donde el cálculo fue de $22.7 \%$ y clasificada en un grado moderado de severidad. Lo anterior se debió posiblemente al bajo número de casos estudiados que favorece el aumento de margen de error, así como el subregistro de tensiones arteriales elevadas por parte de los médicos familiares.

La proporción de pacientes jóvenes con cifras de hipertensión arterial descontrolada es baja, encontrando mayor número de casos en la edad promedio de 61 años, lo cual es coincidente con lo reportado en la literatura, en un estudio de inercia clínica en una unidad de Medicina Familiar de Acapulco Guerrero, donde la edad mínima fue de 37 años y un promedio de 63 años en los pacientes estudiados con esta enfermedad crónica (19).

En la revisión de expedientes donde el médico ha realizado algún cambio al tratamiento antihipertensivo en el manejo de la hipertensión arterial descontrolada se encontró un $66 \%$ en nuestro estudio, comparado con lo realizado con Caro y Colaboradores en el 2002 (20) donde obtuvieron solo $34.7 \%$ y en el 2006 fue de $46.7 \%$ lo cual representa una cifra menor.

Se determinó el número de casos donde no se realiza el ajuste de dosis resultando en un $66.7 \%$ y la congruencia de apego al algoritmo de tratamiento de acuerdo con la guía de práctica clínica en el manejo de hipertensión, pero no se reportan estudios en la literatura donde se haga referencia a este apartado.

\section{CONCLUSIONES}

La inercia terapéutica encontrada tiene una severidad moderada y es importante la capacitación del médico familiar en las guías de práctica clínica y la supervisión estricta de los pacientes con hipertensión descontrolada. 


\section{REFERENCIAS}

1.- Norma Oficial Mexicana NOM-030-SSA2-2009. Para la prevención, detección, diagnóstico, tratamiento y control de la hipertensión arterial sistémica. México. 2009. [consultado Noviembre, 2020] Disponible

En: https://www.cndh.org.mx/sites/default/files/doc/Programas/VIH/Leyes\%20y\%20normas\%20y\%20regla mentos/Norma\%20Oficial\%20Mexicana/NOM-030-SSA2-2009.pdf

2.- Lim SS, Vos T, Flaxman AD, Danaei G, Shibuya K, Adair-Rohani H, et al. A comparative risk assessment of burden of disease and injury attributable to 67 risk factors and risk factor clusters in 21 regions, 1990-2010: a systematic analysis for the Global Burden of Disease Study 2010. Lancet 2012; 380(9859): 2224-60. https://doi.org/10.1016/S0140-6736(12)61766-8

3.- World Health Organization. Chapter 1. Burden: mortality, morbidity and risk factors. En: World Health Organization. Global status report on noncommunicable diseases 2010. Ginebra: WHO, 2011:932 [citado noviembre, 2020]. Disponible en: https://www.who.int/nmh/publications/ncd_report_chapter1.pdf?ua=1

4. Campos-Nonato I, Hernández-Barrera L, Pedroza-Tobías A, Medina C, Barquera S. Hipertensión arterial en adultos mexicanos: prevalencia, diagnóstico y tipo de tratamiento Ensanut MC 2016. Salud Publica Mex. 2018; 60(3): 233-43. https://doi.org/10.21149/8813

5.- Patricia López. Uno de cada cuatro connacionales con hipertensión, 31 millones de mexicanos. Gaceta UNAM. Mayo 20, 2019. [consultado Noviembre, 2020 ] Disponible En:

https://www.gaceta.unam.mx/con-hipertension-31-millones-de-mexicanos/

6.- World Health Organization. A global brief on hypertension. Silent killer, global public health crisis. Ginebra: WHO, 2013 [citado Noviembre, 2020]. Disponible en: https://apps.who.int/iris/bitstream/handle/10665/79059/WHO_DCO_WHD_2013.2_eng.pdf?sequence= 1

7.- Aujoulat I, Jacquemin P, Rietzschel E, Scheen A, Tréfois P, Wens J, Darras E, Hermans MP. Factors associated with clinical inertia: an integrative review. Adv Med Educ Pract. 2014 May 8;5:141-7. doi: 10.2147/AMEP.S59022.

8.-Roa L, Monreal M, Carmona J, Aguilar E, Coll R, Suárez C. Inercia terapéutica en prevención secundaria de enfermedad cardiovascular. Registro frena. Medicina Clínica 2016; 134(2): 57-63.

9.- Giugliano D, Esposito K. Clinical Inertia as a clinical safeguard. JAMA 2011; 305 (15): 1591-1592.

10.- Fine L, Cutler J. Hypertension and the Treating Physician Understanding and Reducing Therapeutic Inertia. Hypertension 2016; 47: 319-320.

11.- James PA, Oparil S, Carter BL, Cushman WC, Dennison-Himmelfarb C, Handler J, et al 2014 evidence-based guideline for the management of high blood pressure in adults; report from the panel members appointed to the Eighth JOINT National Committee (JNC 8) JAMA 2014; 311 (5):507-20.

12.- Barrios V, Escobar C. Utilidad de la terapia combinada en la prevención y tratamiento de la enfermedad cardiovascular. Rev Clin Esp. 2010; 210(5): 230-236

13.- Redon J, Coca A, Lázaro P, Dolores M, Cabañas M, Gil N et al. Factors associated with therapeutic inertia in hypertension: validation of a predictive model. Journal of Hypertension 2015; 28: 1770-1777. 
14.- Butler J, Papadimitriou L, Georgiopoulou V, Skopicki H, Dunbar S, Kalogeropoulos A. Comparing Sodium Intake Strategies in Heart Failure: Rationale and Design of the Prevent Adverse Outcomes in Heart Failure by Limiting Sodium (PROHIBIT) Study. Circ Heart Fail. 2015 May;8(3):636-45. doi: 10.1161/CIRCHEARTFAILURE.114.001700.

15.- Whelton PK, Carey RM, Aronow WS, Casey DE Jr, Collins KJ, Dennison Himmelfarb C, DePalma SM, Gidding S, Jamerson KA, Jones DW, MacLaughlin EJ, Muntner P, Ovbiagele B, Smith SC Jr, Spencer CC, Stafford RS, Taler SJ, Thomas RJ, Williams KA Sr, Williamson JD, Wright JT Jr. 2017 ACC/AHA/AAPA/ABC/ACPM/AGS/APhA/ASH/ASPC/NMA/PCNA Guideline for the Prevention, Detection, Evaluation, and Management of High Blood Pressure in Adults: A Report of the American College of Cardiology/American Heart Association Task Force on Clinical Practice Guidelines. Hypertension. 2018 Jun; 71(6):e13-e115. doi: 10.1161/HYP.0000000000000065. Epub 2017 Nov 13. Erratum in: Hypertension. 2018 Jun;71(6):e140-e144.

16.- Diagnóstico y Tratamiento de la Hipertensión Arterial en el Primer nivel de Atención, México: Instituto Mexicano del Seguro Social; 08/07/2014. [consultado Noviembre, 2020 ] Disponible En: http://cenetec-difusion.com/gpc-sns/?page_id=5223

17.- O'Connor PJ, Sperl-Hillen JAM, Johnson PE, et al. Clinical Inertia and Outpatient Medical Errors. In: Henriksen K, Battles JB, Marks ES, et al., editors. Advances in Patient Safety: From Research to Implementation (Volume 2: Concepts and Methodology). Rockville (MD): Agency for Healthcare Research and Quality (US); 2005 Feb. Available from: https://www.ncbi.nlm.nih.gov/books/NBK20513/

18.-Pallarés V, Pascual R. Non-compliance and therapeutic inertia: two unanswered questions in clinical practice. Curr Med Research Op 2014; 30 (5): 839-840.

19.- García G, Reyes M. Inercia clínica en pacientes con Diabetes Mellitus en una Unidad de Medicina Familiar de Acapulco Guerrero. Aten Fam 2017; 4 (3): 102-106

20.- Caro JL et al. Control de la presión arterial en la población hipertensa española atendida en atención primaria. Estudio PRESCAP 2006. Medicina Clínica 2008;130(18): 681-687 